\title{
Study of a Fractal-Fractional Smoking Models with Relapse and Harmonic Mean Type Incidence Rate
}

\author{
Zareen A. Khan $\mathbb{D}^{1},{ }^{1}$ Mati ur Rahman, ${ }^{2}$ and Kamal Shah $\mathbb{D}^{3,4}$ \\ ${ }^{1}$ Department of Mathematics, College of Science, Princess Nourah bint Abdulrahman University, Riyadh, Saudi Arabia \\ ${ }^{2}$ Department of Mathematics, Shanghai Jiao Tong University, 800 Dongchuan Road, Shanghai, China \\ ${ }^{3}$ Department of Mathematics, University of Malakand, Chakdara Dir(L), 18000 Khyber Pakhtunkhwa, Pakistan \\ ${ }^{4}$ Department of Mathematics and General Sciences, Prince Sultan University, Riyadh, Saudi Arabia
}

Correspondence should be addressed to Zareen A. Khan; zakhan@pnu.edu.sa

Received 24 June 2021; Accepted 24 September 2021; Published 11 October 2021

Academic Editor: Richard I. Avery

Copyright (c) 2021 Zareen A. Khan et al. This is an open access article distributed under the Creative Commons Attribution License, which permits unrestricted use, distribution, and reproduction in any medium, provided the original work is properly cited.

\begin{abstract}
This manuscript investigates fractal-fractional order smoking models with relapse and harmonic mean type incidence rate under the Caputo derivative. We derive the existence and unique results about the solution for the considered model via fixed point theory. For the stability of the considered system, Ulam-Hyers (UH) approach is used. We compute the numerical solution by using fractional Adams-Bashforth method. For the simulation of the model, we consider different values of fractional order $\delta$ and fractal dimension $\theta$ by using some real values of the parameters. The proposed scheme is used to simulate the available data for some smoking community including potential, light, and quit smokers. Various graphical presentations are given to understand the dynamics of the model at various fractional orders.
\end{abstract}

\section{Introduction}

The first biological model that describes the dynamics of infectious disease was presented in 1927. Later on, scientists and researchers started to investigate different properties of the models such as the spreading behavior and trends of the diseases by studying the various aspects [1-4]. They have formulated several models for different diseases like pine wilt, HIV, viral disease including leishmania, TB, and COVID-19 [5-12].

Smoking is also similar to infectious diseases by spreading its behavior in the population. The ratio of diseases due to smoking is increasing day by day. Castillo-Garsow et al. [13] formulated for the first time a simple giving up smoking model with known spreading behavior of smoking in the community. The same authors modified and extended the work by adding another class of light smoking. The authors [14] focused on the control strategy of smoking epidemic by choosing optimal campaigns. Furthermore, some of the smokers may relapse because they may have frequent contacts with smokers, whereas some of them may cease smoking permanently. Rahman et al. [15] have been worked on a smoking model and included the relapse terms for the quit smokers.

The abovementioned models have been investigated under ordinary derivatives. During the last twenty years, fractional calculus (FC) has gained more interest from the researcher and been used in different fields of sciences. Mathematical models along with fractional differential equations (FDEs) have been proved for several smoking models. Compared with integer-order model, fractional-order models have better fitting degree with different experimental results in signal processing, mathematical biology and engineering [16, 17-19]. In this regard, Mahdy et al. [20] found the approximate solution for a smoking model by utilizing the Sumudu transform with Caputo derivative. Sing et al. [21] has been introduced a giving up smoking dynamic fractional model with nonsingular kernel. Khan et al. [22] have been studied a biological model of smoking type with some iterative method. Mohamed et al. [23] used reduced differential transform method to solve the nonlinear smoking 
fractional-order model. Alrabaiah et al. [24] have been applied Adams-Bashforth-Moulton method to investigate the tobacco smoking fractional model order containing snuffing class. Therefore, for the past periods, to develop the real phenomena for a better degree of precision and accuracy, FDEs have been utilized very well. Many researchers have utilized several methods for studying the theoretical investigation of fractional-order mathematical models, (for instance see [25-29]). For further detail, see [30-35]. Adomian in 1980 introduced a useful decomposition method for the solution of nonlinear systems analytically. Later on, the abovementioned method has been slowly enforced as an actual tool for consideration semianalytical or estimated results to several systems of applied sciences. Mathematical models have been examined widely using the Homotopy method, decomposition method along with integral transforms, and difference methods, for details, see $[30,31]$. Recently, many methods have been utilized to handle problems of fractional order (see details in [36-38]).

Keeping in mind that derivative of noninteger can be defined in several ways. The first definition of fractional derivative was given by Riemann-Liouville. Later on in 1967, Caputo gave his own definition which has been increasingly used. The mentioned both definitions include singular kernels which often cause problem in numerical investigations. To overcome these difficulties, recently, Caputo and Fabrizio [39] have introduced a new definition. The said definition contains exponential function instead of singular kernel. In subsequent years, the said definition has been further generalized by Atangana and Baleanu [40] by replacing exponential function on Mittag-Leffler one. This fact has been proved that the concerned derivative also has interesting features (see [41-46]).

Recently, the area involves fractal-fractional derivative has got much attention (see [47, 49-51]). Motivated from the above work and from, we consider the model presented in [48] to fractal-fractional (FF) order in sense of Caputo operator which has various advantages. This model consists of four compartments, namely, people vulnerable to smoking $P(t)$, light smokers $L(t)$, smoker class $S(t)$, and quit smokers $Q(t)$. This work also includes theoretical, practical analytical, and numerical results of smoking models with relapse and harmonic mean type incidence rate. Our considered model under Caputo operator for fractional-order $\delta$ and fractal dimension $\theta$ is as follows:

$$
\begin{aligned}
{ }^{F F} \mathbf{D}^{\delta, \theta} P & =\prod-2 \beta \frac{P(t) L(t)}{P(t)+L(t)}-(b+\alpha) P(t)+\tau Q(t), \\
{ }^{F F} \mathbf{D}^{\delta, \theta} L & =2 \beta \frac{P(t) L(t)}{P(t)+L(t)}-(b+\alpha+\zeta) L(t), \\
{ }^{F F} \mathbf{D}^{\delta, \theta} S & =\zeta L(t)-(b+\alpha+\phi) S(t), \\
{ }^{F F} \mathbf{D}^{\delta, \theta} Q & =\phi S(t)-(b+\alpha+\tau) Q(t),
\end{aligned}
$$

with initial conditions

$$
P(0)=P_{0}, L(0)=L_{0}, S(0)=S_{0}, Q(0)=Q_{0},
$$

where $\beta$ is the transmission rate that the potential smoker contact with the chain smoker, $\tau$ is the relapse rate, $\Pi$ is the recruitment rate, $\alpha$ is the natural death rate, and $b$ is the death rate induced by smoking. Also, $\zeta$ is the conversion rate from light to chain smoker class. In same line, $\varphi$ is the chain smokers rate when they quit smoking. We also discuss some stability results devoted to $\mathrm{UH}$ type. The mentioned stability has been recently investigated for various problems of FDEs (see [55-57]).

The rest of the paper we organized is as follows: Section 2 is related to basic definitions and theorems. By using fixed point theorems, we show some suitable results for the uniqueness and existence in Section 3. With the help of famous $A B$ technique, we find the numerical solution of the considered system in Section 4. Using the $\mathrm{AB}$ technique, we also perform the numerical simulation by using Matlab for getting the graphical representation for our analytic and briefly discuss the obtained results. Finally, we conclude our work in Section 5.

\section{Basic Results}

Definition 1 (see [47]). Let $\boldsymbol{U}(t)$ on $a<t<b$ be a continuous and differentiable function with order $\theta$, then the $F F$ order derivative can be defined as

$$
{ }^{F F} \mathbf{D}_{t}^{\delta, \theta}(\boldsymbol{U}(t))=\frac{1}{(p-\delta)} \frac{d}{d t^{\theta}} \int_{0}^{t}(t-x)^{p-\delta-1} \boldsymbol{U}(x) d x
$$

along-with $p-1<\delta, \theta \leq p$, where $p \in N$ and $d \boldsymbol{U}(x) / d x^{\theta}=$ $\lim _{t \rightarrow 0} \boldsymbol{U}(t)-\boldsymbol{U}(x) / t^{\theta}-x^{\theta}$.

Definition 2 (see [47]). Let $\boldsymbol{U}(t)$ be continuous on $a<t<b$ then the $F F$ order integral of $\boldsymbol{U}(t)$ with order $\delta$ is defined as

$$
{ }^{F F} \mathbf{I}^{\delta} \boldsymbol{U}(t)=\frac{\theta}{\Gamma(\delta)} \int_{0}^{t}(t-x)^{\delta-1} x^{\theta-1} \boldsymbol{U}(x) d x . .
$$

Definition 3. The system (1) is $U H$ stable if $\exists$ any real number $C_{\delta, \theta} \geq 0$ such that for every $\epsilon>0$ and all the solutions $\mathscr{W} \in C^{1}([0, T], R)$, where $0<t<T<\infty$, the inequality can be defined as

$$
\left|{ }^{F F} \mathbf{D}^{\delta, \theta} \mathscr{W}(t)-\Psi(t, \mathscr{W}(t))\right| \leq \epsilon, t \in[0, T]
$$

$\mathscr{Y} \in C^{1}([0, T], R)$ is the unique solution for the considered model (1) such that

$$
|\mathscr{W}(t)-\mathscr{Y}(t)| \leq C_{\delta, \theta}, t \in[0, T] .
$$

Note: let us define a Banach space For the qualitative analysis $U=X \times X \times X \times X$, where $X=C([0, T])$ with norm: $\|\mathscr{W}\|=\|\mathrm{P}, \mathrm{L}, \mathrm{S}, \mathrm{Q}\|=\max _{t \in[0, T]}\{|P(t)|+|L(t)|+|S(t)|+|Q(t)|\}$. 


\section{Theoretical Results of Model (1)}

Here in this section, we will investigate the model (1) for existence. Since the given integral is differentiable, so we can express the RHS of the model (1) as

$$
\begin{aligned}
& { }^{\mathscr{L} \mathscr{L}} \mathbf{D}^{\delta} P(t)=\theta t^{\theta-1} G_{1}(P, L, S, Q, t)=\prod-2 \beta \frac{P(t) L(t)}{P(t)+L(t)}-(b+\alpha) P(t)+\tau Q(t), \\
& \mathscr{R L} \mathbf{D}^{\delta} L(t)=\theta t^{\theta-1} G_{2}(P, L, S, Q, t)=2 \beta \frac{P(t) L(t)}{P(t)+L(t)}-(b+\alpha+\zeta) L(t), \\
& \mathscr{R} \mathscr{L} \mathbf{D}^{\delta} S(t)=\theta t^{\theta-1} G_{3}(P, L, S, Q, t)=\zeta L(t)-(b+\alpha+\varphi) S(t), \\
& \mathscr{R} \mathscr{L} \mathbf{D}^{\delta} Q(t)=\theta t^{\theta-1} G_{4}(P, L, S, Q, t)=\varphi S(t)-(b+\alpha+\tau) Q(t) .
\end{aligned}
$$

In view of (7) and for $t \in[0, T]$, the proposed model may be written in the following form

$$
\begin{aligned}
{ }^{\mathscr{L}} \mathbf{D}^{\delta} \mathscr{W}(t) & =\theta t^{\theta-1} \Psi(t, \mathscr{W}(t)), 0<\delta, \theta \leq 1, \\
\mathscr{W}(0) & =\mathscr{W}_{0},
\end{aligned}
$$

by changing ${ }^{\mathscr{R} \mathscr{L}} \mathbf{D}^{\delta, \theta}$ with ${ }^{C} \mathbf{D}^{\delta, \theta}$ and using the integral of Riemann-Liouville, the solution of (8) will be

$$
\mathscr{W}(t)=\mathscr{W}_{0}+\frac{\theta}{\Gamma(\delta)} \int_{0}^{t} x^{\theta-1}(t-x)^{\delta-1} \Psi(x, \mathscr{W}(x)) d x
$$

where

$\mathscr{W}(t)=\left\{\begin{array}{l}\mathrm{P}(t) \\ \mathrm{L}(t) \\ \mathrm{S}(t) \\ \mathrm{Q}(t),\end{array}, \mathscr{W}_{0}(t)=\left\{\begin{array}{l}\mathrm{P}_{0} \\ \mathrm{~L}_{0} \\ \mathrm{~S}_{0} \\ \mathrm{Q}_{0},\end{array}, \Psi(t, \mathscr{W}(t))=\left\{\begin{array}{l}\mathrm{G}_{4}(\mathrm{P}, \mathrm{L}, \mathrm{S}, \mathrm{Q}, t), \\ \mathrm{G}_{4}(\mathrm{P}, \mathrm{L}, \mathrm{S}, \mathrm{Q}, t), \\ \mathrm{G}_{4}(\mathrm{P}, \mathrm{L}, \mathrm{S}, \mathrm{Q}, t), \\ \mathrm{G}_{4}(\mathrm{P}, \mathrm{L}, \mathrm{S}, \mathrm{Q}, t) .\end{array}\right.\right.\right.$

Now, if we transform (1) to fixed point problem and let the operator $\mathscr{T}: \mathbf{V} \longrightarrow \mathbf{V}$ can be defined by

$$
\mathscr{T}(\mathscr{W})(\mathrm{t})=\mathscr{W}_{0}(\mathrm{t})+\frac{\theta}{\Gamma(\delta)} \int_{0}^{t} x^{\theta-1}(t-x)^{\delta-1} \Psi(x, \mathscr{W}(x)) d x
$$

To find the existence results of the considered model, we use the following theorem [54].

Theorem 4. If the operator $\mathscr{T}: \mathbf{V} \longrightarrow \mathbf{V}$ be completely continuous and the set

$$
\mathscr{J}(\mathscr{T})=\{\mathscr{W} \in \mathbf{V}: \mathscr{W}=v \mathscr{T}(\mathscr{W}), v \in[0,1]\}
$$

is bounded, then, the operator $\mathscr{T}$ has at least one fixed point in $\mathbf{V}$.

Theorem 5. Suppose the operator $\Psi: \mathscr{D} \times V \longrightarrow R$ is a continuous operator. Then, $\mathscr{T}$ is compact.
Proof. First, we will show that $\mathscr{T}: \mathbf{V} \longrightarrow \mathbf{V}$ which is defined in (12) is continuous. Consider $\mathscr{B}$ is a bounded set in $\mathbf{V}$, then $\exists C_{\Psi}>0$ with $|\Psi(t, \mathscr{W}(t))| \leq C_{\Psi}$, for all $\mathscr{W} \in B$. Any $\mathscr{W} \in B$, we have

$$
\begin{aligned}
\|\mathscr{T}(\mathscr{W})\| & \leq \frac{\theta \mathscr{C}_{\Psi}}{\Gamma(\delta)} \max _{t \in[0, T]}\left|\int_{0}^{t}(\tau-x)^{\delta-1} x^{\theta-1} d x\right| \\
& \leq \frac{\theta \mathscr{C}_{\Psi}}{\Gamma(\delta)} \max _{t \in[0, T]} \int_{0}^{t}(1-z)^{\theta-1} z^{\delta-1} t^{\delta+\theta-1} d z \\
& \leq \frac{\theta \mathscr{C}_{\Psi} T^{\delta+\theta-1}}{\Gamma(\delta)} \mathrm{B}(\delta, \theta) .
\end{aligned}
$$

Hence, (14) implies that $\mathscr{T}$ is uniformly bounded, where Beta function can be written as $B(\delta, \theta)$. Further, for equicontinuity of the operator $\mathscr{T}$, for any $t_{1}, t_{2} \in D$ and $\mathscr{W} \in B$, we obtain

$$
\begin{aligned}
\left\|\mathscr{T}\left(\mathscr{W}\left(t_{1}\right)+\right)-\mathscr{T}\left(\mathscr{W}\left(t_{2}\right)\right)\right\| \leq & \frac{\theta \mathscr{C}_{\Psi}}{\Gamma(\delta)} \max _{t \in[0, T]} \mid \int_{0}^{t_{1}}\left(t_{1}-x\right)^{\delta-1} x^{\theta-1} d x \\
& -\int_{0}^{t_{2}}\left(t_{2}-x\right)^{\delta-1} x^{\theta-1} d x \mid \\
& \leq \frac{\theta \mathscr{C}_{\Psi} \mathrm{B}(\delta, \theta)}{\Gamma(\delta)}\left(t_{1}^{\delta+\theta-1}-t_{2}^{\delta+\theta-1}\right) \\
& \longrightarrow 0 \text { as } t_{1} \longrightarrow t_{2} .
\end{aligned}
$$

Hence, $\mathscr{T}$ is equi-continuous and then the operator $\mathscr{T}$ is bounded and continuous as well, therefore, by Arzelá-Ascoli theorem, the operator $\mathscr{T}$ is relatively compact and so completely continuous. Furthermore, we use the following hypothesis:

(C) There exist constants $L_{\Psi}>0$ such that, for each $\mathscr{W}$, $\overline{\mathscr{W}} \in f$, we have

$$
|\Psi(t, \mathscr{W})-\Psi(t, \overline{\mathscr{W}})| \leq \mathrm{L}_{\Psi}|\mathscr{W}|-\overline{\mathscr{W}} \mid
$$

For existence uniqueness, we use fixed point approach as given in [54].

Theorem 6. Applying the hypothesis (C) and if $\Theta<1$, then, the model (1) has a unique solution if

$$
\Theta=\frac{\theta L_{\Psi} T^{\delta+\theta-1}}{\Gamma(\delta)} B(\delta, \theta)<1
$$

Proof. Assume $\max _{t \in[0, T]}|\Psi(t, 0)|=\mathscr{K}_{\Psi}<\infty$, such that

$$
r \geq \frac{\theta \mathrm{T}^{\delta+\theta-1} \mathrm{~B}(\delta, \theta) \mathscr{K}_{\Psi}}{\Gamma(\delta)-\theta \mathrm{T}^{\delta+\theta-1} \mathrm{~B}(\delta, \theta) \mathrm{L}_{\Psi}} .
$$


We prove that $\mathscr{T}(\mathscr{B} r) \subset \mathscr{B} r$, where $B_{r}=\{W \in f:\|W\|$ $\leq r\}$ and $\mathscr{W} \in B_{r}$, we have

$$
\begin{aligned}
\|\mathscr{T}(\mathscr{W})\| & \leq \frac{\theta}{\Gamma(\delta)} \max _{t \in[0, T]} \int_{0}^{t} x^{\theta-1}(t-x)^{\delta-1}(|\Psi(t, \mathscr{W}(t))-\Psi(t, 0)|+|\Psi(t, 0)|) d x \\
& \leq \frac{\theta \mathrm{T}^{\delta+\theta-1} \mathrm{~B}(\delta, \theta)\left(\mathrm{L}_{\Psi}\|\mathscr{W}\|+\mathscr{K}_{\Psi}\right)}{\Gamma(\delta)}, \\
& \leq \frac{\theta \mathrm{T}^{\delta+\theta-1} \mathrm{~B}(\delta, \theta)\left(\mathrm{L}_{\Psi} r+\mathscr{K}_{\Psi}\right)}{\Gamma(\delta)}, \leq r .
\end{aligned}
$$

Suppose the operator $\mathscr{T}: \mathbf{V} \longrightarrow \mathbf{V}$ is defined in (12). Using the assumption $\mathbf{C}$ and for every $t \in \mathscr{D}, \mathscr{W}, \overline{\mathscr{W}} \in \mathscr{D}$, we obtain

$$
\begin{aligned}
\|\mathscr{T}(\mathscr{W})-\mathscr{T}(\overline{\mathscr{W}})\| \leq & \frac{\theta}{\Gamma(\delta)} \max _{t \in[0, T]} \mid \int_{0}^{t} x^{\theta-1}(t-x)^{\delta-1} \Psi(x, \mathscr{W}(x)) d x \\
& -\int_{0}^{t} x^{\theta-1}(t-x)^{\delta-1} \Psi(x, \overline{\mathscr{W}}(x)) d x \mid, \\
\leq & \Theta\|\mathscr{W}-\overline{\mathscr{W}}\| .
\end{aligned}
$$

By this, $\mathscr{T}$ is contraction by using (20). Therefore, equation (10) has one solution and so our model (1) has unique solution.

Now, we have to develop UH stability for the considered system (1), taking $\psi \in C(D)$ depending on the solution with $\psi(0)=0$. Then

(i) $|\psi(t)| \leq \varepsilon$, for $\varepsilon>0$

(ii) ${ }^{F F} D_{t}^{\delta, \theta} \mathscr{W}(t)=\Psi(t, \mathscr{W}(t))+\psi(t)$

Lemma 7. The solution of perturbed equation

$$
\begin{aligned}
{ }^{F F} \mathbf{D}^{\delta, \theta} \mathscr{W}(t) & =\Psi(t, \mathscr{W}(t))+\psi(t), \\
\mathscr{W}(0) & =\mathscr{W}_{0},
\end{aligned}
$$

satisfies the given relation

$$
\begin{aligned}
\mid \mathscr{W}(t) & -\left(\mathscr{W}_{0}(t)+\frac{\theta}{\Gamma(\delta)} \int_{0}^{t} x^{\theta-1}(t-s)^{\delta-1} \Psi(x, \mathscr{W}(x))\right) \mid \\
& \leq\left(\frac{\theta T^{\delta+\theta-1} B(\delta, \theta)}{\Gamma(\delta)}\right) \varepsilon=C_{\delta, \theta} \mathcal{\varepsilon} .
\end{aligned}
$$

Theorem 8. With the assumption (C) and (22), the solution of the integral equation (1) is UH stable. Hence, the analytical results of the considered system are $U H$ stable if $\Theta<1$, where $\Theta$ is given in (17).
TABle 1: Parametric values for our model (1).

\begin{tabular}{lcc}
\hline Parameter & Value & Source \\
\hline$\Pi$ & 10.25 & Assumed \\
$\beta$ & 0.038 & {$[52]$} \\
$b$ & 0.0019 & {$[52,53]$} \\
$\alpha$ & 0.0111 & {$[52]$} \\
$\zeta$ & 0.021 & {$[15,52]$} \\
$\varphi$ & 0.000274 & {$[15]$} \\
$\tau$ & 0.006 & Assumed \\
\hline
\end{tabular}

Proof. Suppose that $\mathscr{Z} \in \mathbf{V}$ be a unique solution and $\mathscr{W} \in \mathbf{V}$ be any solution of (10), then using fractalfractional integration as in an equation (2), we have

$$
\begin{aligned}
|\mathscr{W}(t)-\mathscr{E}(t)|= & \left|\mathscr{W}(t)-\left(\mathscr{Z}_{0}(t)+\frac{\theta}{\Gamma(\delta)} \int_{0}^{t}(t-x)^{\delta-1} x^{\theta-1} \Psi(x, \mathscr{Z}(x)) d x\right)\right|, \\
\leq & \left|\mathscr{W}(t)-\left(\mathscr{W}_{0}(t)+\frac{\theta}{\Gamma(\delta)} \int_{0}^{t}(t-x)^{\delta-1} x^{\theta-1} \Psi(x, \mathscr{W}(x)) d x\right)\right|, \\
& +\mid\left(\mathscr{W}_{0}(t)+\frac{\theta}{\Gamma(\delta)} \int_{0}^{t}(t-x)^{\delta-1} x^{\theta-1} \Psi(x, \mathscr{W}(x)) d x\right) \\
& -\left(\mathscr{Z}_{0}(t)+\frac{\theta}{\Gamma(\delta)} \int_{0}^{t}(t-x)^{\delta-1} x^{\theta-1} \Psi(x, \mathscr{Z}(x)) d x\right) \mid, \\
\leq & \mathrm{C}_{\delta, \theta} \mathcal{E}+\frac{\theta \mathrm{T}^{\delta+\theta-1} \mathrm{~L}_{\mathscr{W}}}{\Gamma(\delta)} \mathrm{B}(\delta, \theta)\|\mathscr{W}-\mathscr{Z}\|, \\
\leq & \mathrm{C}_{\delta, \theta}+\Theta\|\mathscr{W}-\mathscr{Z}\| .
\end{aligned}
$$

Which we have

$$
\|\mathscr{W}-\mathscr{Z}\| \leq \mathrm{C}_{\delta, \theta}+\Theta\|\mathscr{W}-\mathscr{Z}\|
$$

From (24), we can write as

$$
\|\mathscr{W}-\mathscr{Z}\| \leq\left(\frac{\mathrm{C}_{\delta, \theta}}{1-\Theta}\right) \varepsilon .
$$

Thus, from the (25), we conclude that the solution of (10) is UH stable and therefore the proposed model (1) solution is UH stable.

\section{Numerical Scheme}

In this part of the paper, we are constructing the numerical algorithm for the considered model to perform numerical simulation. Here, for numerical method, the construction of equation (10) of the considered model goes to the following form 

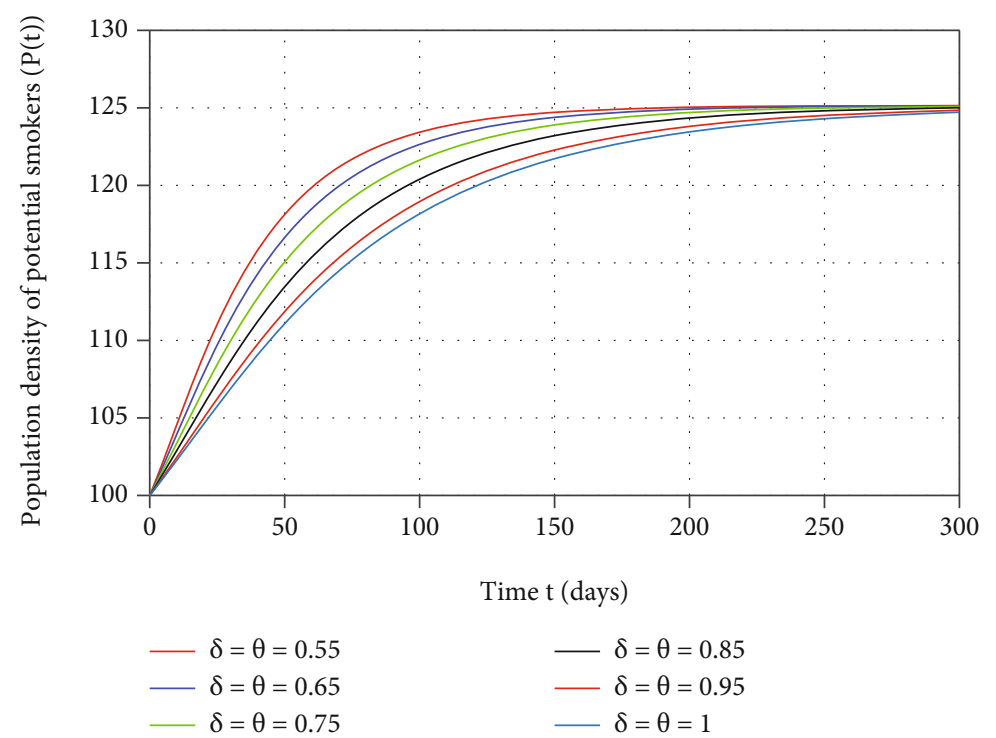

(a)
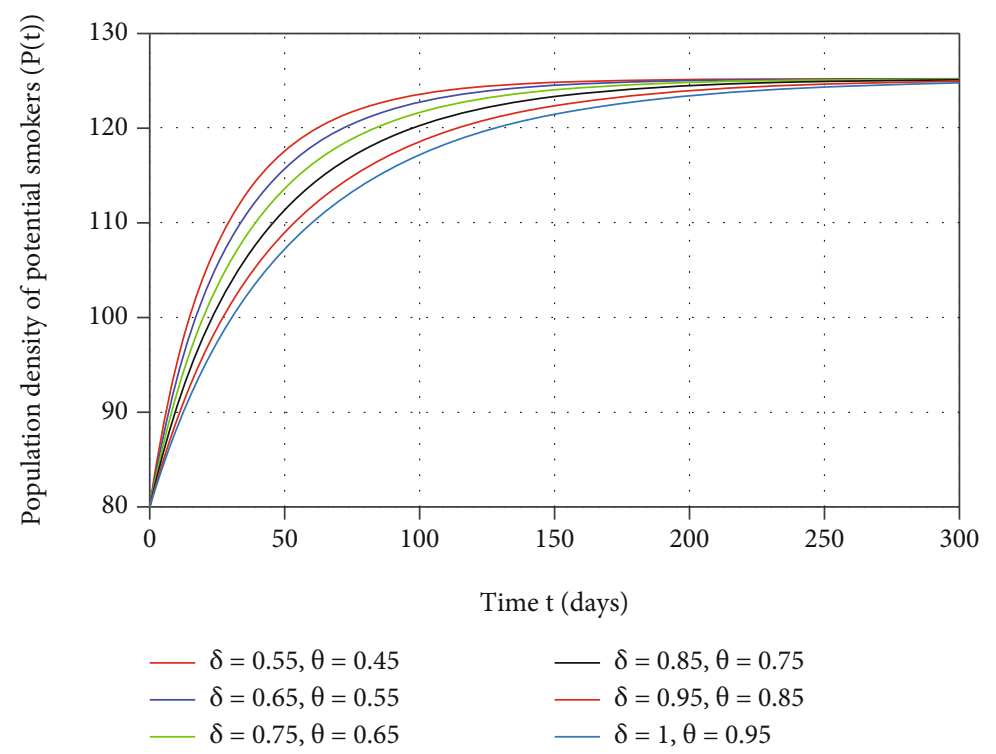

(b)

Figure 1: Graphical representation of potential smokers $(P(t))$ having two different initial values of $P_{0}=100,80$ in the model under investigation (1) at different arbitrary fractal dimension and fractional orders.

$$
\begin{aligned}
& P(t)=P(0)+\frac{\theta}{\Gamma(\delta)} \int_{0}^{t} x^{\theta-1}(t-x)^{\delta-1} G_{1}(P, L, S, Q, x) d x, \\
& L(t)=L(0)+\frac{\theta}{\Gamma(\delta)} \int_{0}^{t} x^{\theta-1}(t-x)^{\delta-1} G_{2}(P, L, S, Q, x) d x, \\
& S(t)=S(0)+\frac{\theta}{\Gamma(\delta)} \int_{0}^{t} x^{\theta-1}(t-x)^{\delta-1} G_{3}(P, L, S, Q, x) d x, \\
& Q(t)=Q(0)+\frac{\theta}{\Gamma(\delta)} \int_{0}^{t} x^{\theta-1}(t-x)^{\delta-1} G_{4}(P, L, S, Q, x) d x .
\end{aligned}
$$

(26) and using the new approach $t_{k+1}$. The first equation of the above system becomes

$$
P_{k+1}=P_{0}+\frac{\theta}{\Gamma(\delta)} \int_{0}^{t_{k+1}} x^{\theta-1}\left(t_{k+1}-x\right)^{\delta-1} G_{1}(P, L, S, Q, x) d x
$$

We obtained the approximate integral from the above equation as

$$
P_{k+1}=P_{0}+\frac{\theta}{\Gamma(\delta)} \sum_{j=0}^{k} \int_{t_{j}}^{t_{j+1}} y^{\theta-1}\left(t_{k+1}-x\right)^{\delta-1} G_{1}(P, L, S, Q, x) d x
$$

Now, we are presenting the numerical solution to the 

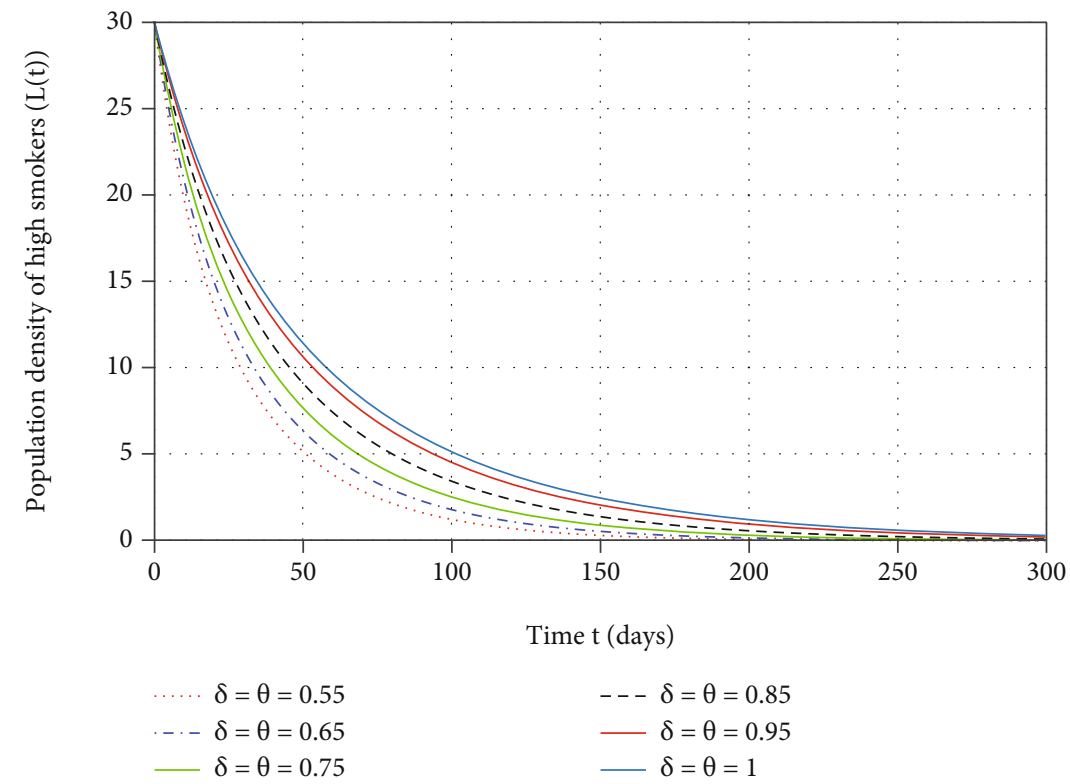

(a)

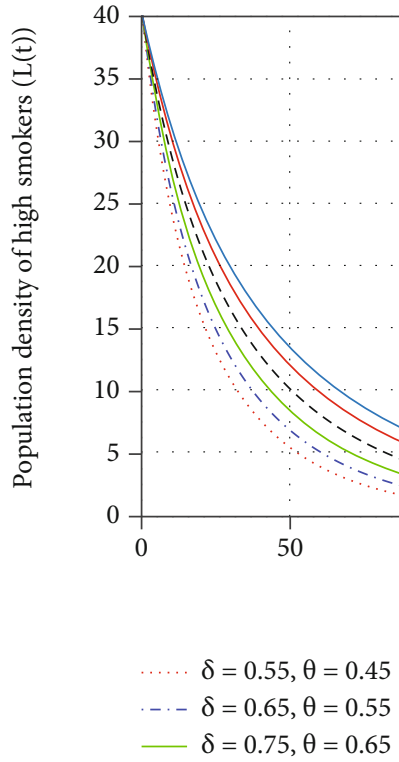




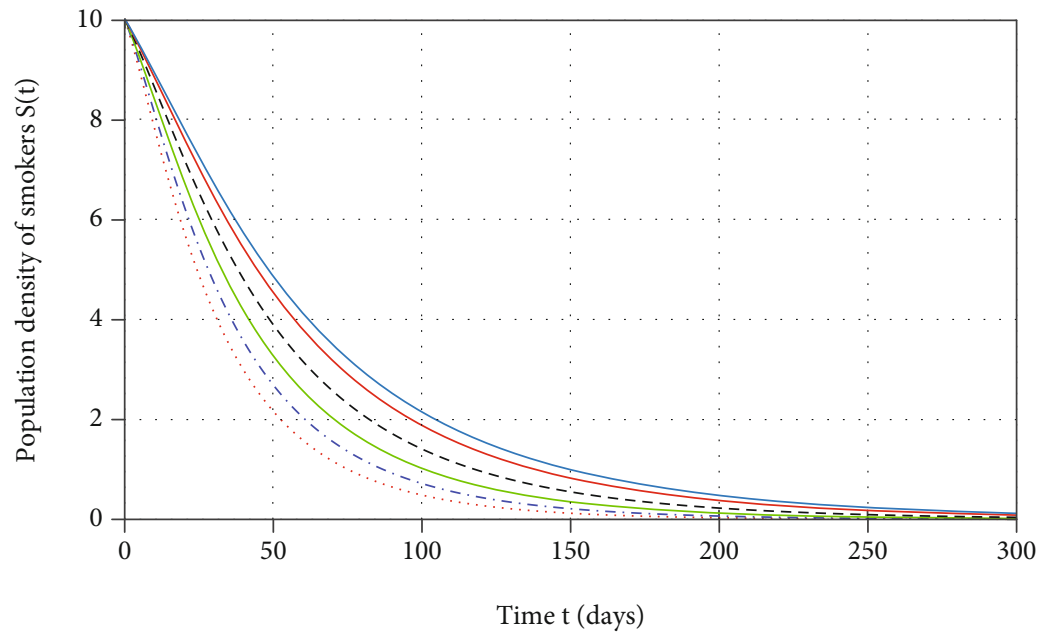

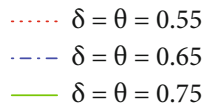

$\delta=\theta=0.75$

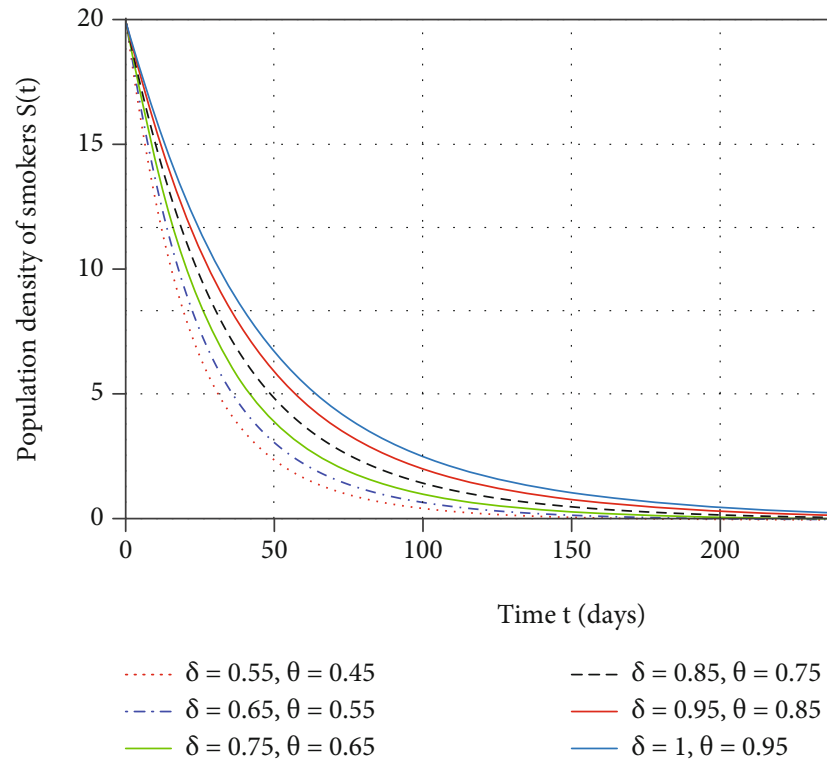

(b)

FiguRE 3: Graphical representation of smokers $(S(t))$ having two different initial values of $L_{0}=10,20$ in the model under investigation (1) at different arbitrary fractal dimension and fractional orders.

Similarly, the remaining terms can be written as

$$
\begin{aligned}
L_{k+1}= & L_{0}+\frac{\theta \hbar^{\delta}}{\Gamma(\delta+2)} \sum_{j=0}^{k}\left[t_{j}^{\theta-1} G_{2}\left(P_{j}, L_{j}, S_{j}, Q_{j}, t_{j}\right)\right. \\
& \times\left((k+1-j)^{\theta}(k-j+2+\theta)-(k-j)^{\theta}(k-j+2+2 \theta)\right) \\
& -t_{j-1}^{\theta-1} G_{2}\left(P_{j-1}, L_{j-1}, S_{j-1}, Q_{j-1}, t_{j-1}\right) \\
& \left.\times\left((k+1-j)^{\theta}+1-(k-j)^{\theta}(k-j+1+\theta)\right)\right], \\
S_{k+1}= & S_{0}+\frac{\theta \hbar^{\delta}}{\Gamma(\delta+2)} \sum_{j=0}^{k}\left[t_{j}^{\theta-1} G_{3}\left(P_{j}, L_{j}, S_{j}, Q_{j}, t_{j}\right)\right. \\
& \times\left((k+1-j)^{\theta}(k-j+2+\theta)-(k-j)^{\theta}(k-j+2+2 \theta)\right)
\end{aligned}
$$

$$
\begin{aligned}
& -t_{j-1}^{\theta-1} G_{3}\left(P_{j-1}, L_{j-1}, S_{j-1}, Q_{j-1}, t_{j-1}\right) \\
& \left.\times\left((k+1-j)^{\theta}+1-(k-j)^{\theta}(k-j+1+\theta)\right)\right], \\
Q_{k+1}= & Q_{0}+\frac{\theta \hbar^{\delta}}{\Gamma(\delta+2)} \sum_{j=0}^{k}\left[t_{j}^{\theta-1} G_{4}\left(P_{j}, L_{j}, S_{j}, Q_{j}, t_{j}\right)\right. \\
& \times\left((k+1-j)^{\theta}(k-j+2+\theta)-(k-j)^{\theta}(k-j+2+2 \theta)\right) \\
& -t_{j-1}^{\theta-1} G_{4}\left(P_{j-1}, L_{j-1}, S_{j-1}, Q_{j-1}, t_{j-1}\right) \\
& \left.\times\left((k+1-j)^{\theta}+1-(k-j)^{\theta}(k-j+1+\theta)\right)\right] .
\end{aligned}
$$




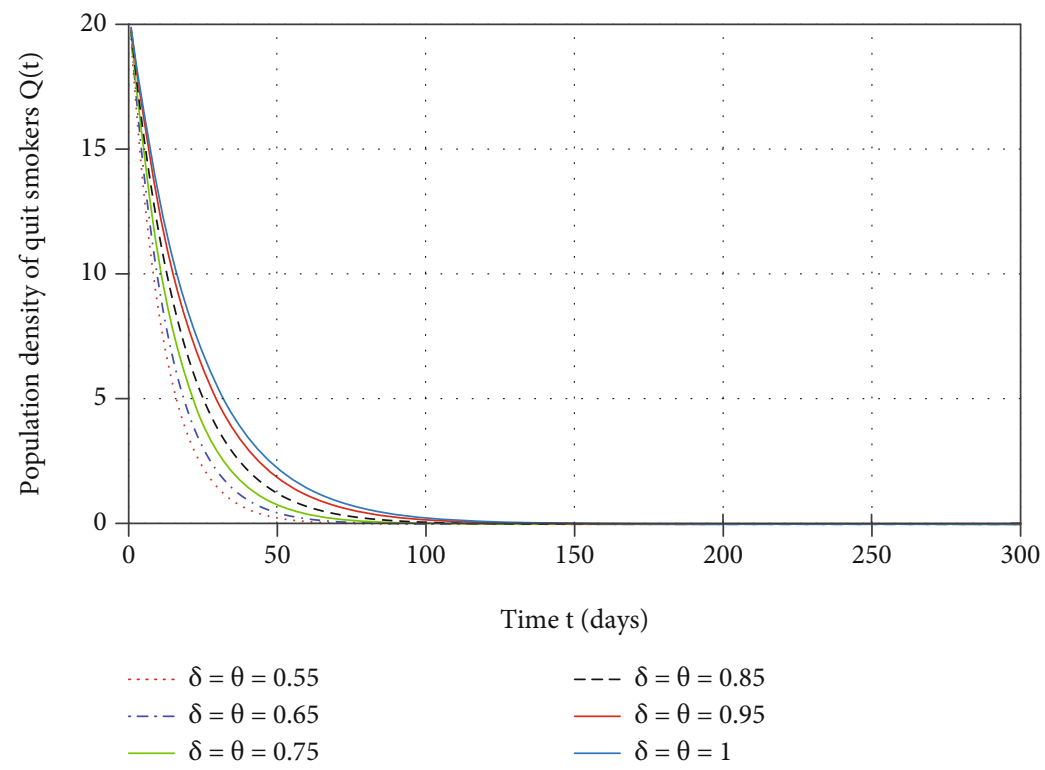

(a)

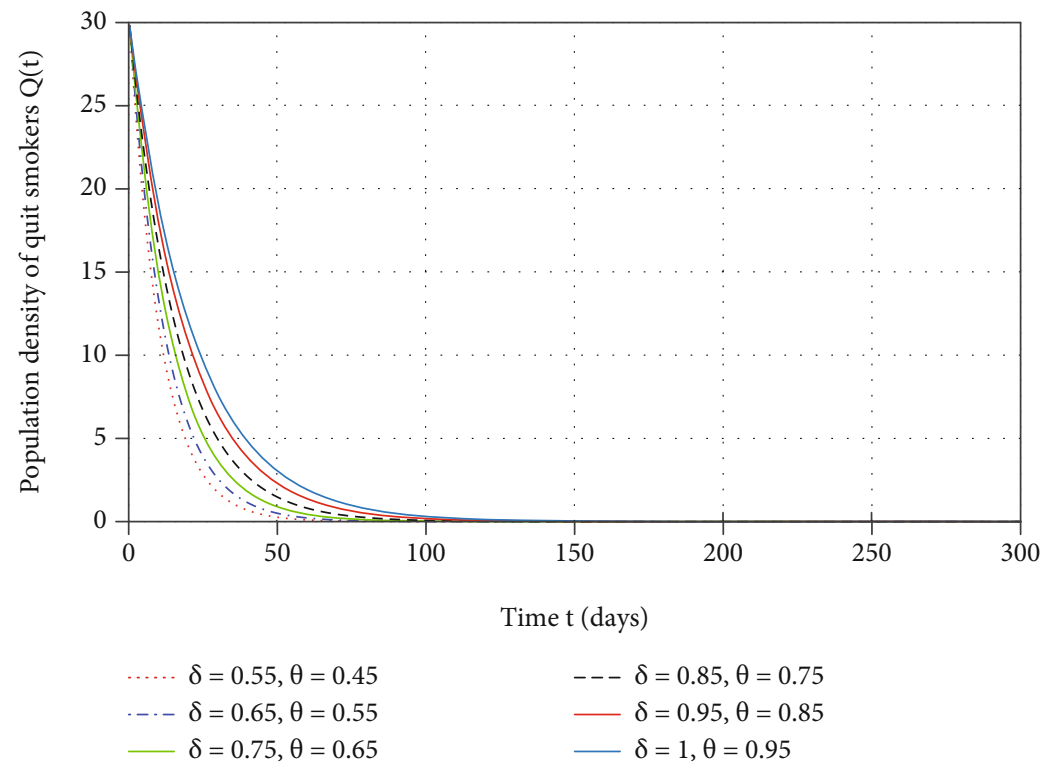

(b)

FIGURE 4: Graphical representation of quit smokers $(Q(t))$ having two different initial values of $L_{0}=20,30$ in the model under investigation (1) at different arbitrary fractal dimension and fractional orders.

4.1. Graphical Representations. In this section, we provide the numerical solution of our proposed model (1) using different values of parameters given in Table 1 for verification of the obtained scheme. We have taken two different sets of initial values of all the compartments in problem (1) for two different fractal dimension $\theta$ and fractional order $\delta$.

Figures 1(a) and 1(b) show the dynamical behavior of potential smoker population $P(t)$ at various fractal dimension $\theta$ and fractional order $\delta$ at two different initial values. On different six fractal-fractional values, the class increases and becomes stable which converges to the same point having two initial values. The increase occurs quickly at high order and slowly at low order and converges to the integer order as we increase the fractional order.
Figures $2(\mathrm{a})$ and $2(\mathrm{~b})$ are the representation of the dynamical behavior of light smokers $L(t)$ at different fractal dimension $\theta$ and fractional order of $\delta$ at two different initial values. On different six fractal-fractional values, the potential smoker class becomes stable and converges to the same point having two initial values.

Figures 3(a) and 3(b) show the dynamical behavior of smokers $S(t)$ at various fractal dimension $\theta$ and fractional order $\delta$ at two different initial values. At six different fractional values, the class decreases and becomes stable which converges to the same converging point for two different initial approximations.

Figures 4(a) and 4(b) show the dynamical behavior of quit smoker $Q(t)$ at various fractal dimension $\theta$ and 
fractional order $\delta$ at two different starting values. On six different fractional values, the class declines quickly nearly at all fractional orders but then becomes stable which converges to the same point having two initial guesses.

\section{Conclusion}

In this manuscript, we have analyzed a giving up smoking model under the concept of fractal-fractional order derivative in Caputo sense. The considered model has been investigated for some theatrical analysis including existence theory and stability results. In this regard, sufficient results have been established for existence and uniqueness of solution by using Banach-contraction and Schauder's theorems of nonlinear functional analysis. The Ulam-Hyers stability analysis has been developed by using the usual nonlinear analysis tools. Further, we have used fractional Adam Bashforth method and developed an algorithm to compute numerical results. We have used various values of fractal dimensions and fractional orders to present the results graphically. From graphical presentation, one can observe that fractal and fractional calculus have the ability to present the dynamics of real-world problems more comprehensively.

\section{Data Availability}

Data sharing is not applicable to this article as no data sets were generated or analyzed during the current study.

\section{Conflicts of Interest}

The authors declare that they have no competing interests.

\section{Authors' Contributions}

All authors read and approved the final manuscript.

\section{Acknowledgments}

This research was funded by the Deanship of Scientific Research at Princess Nourah bint Abdulrahman University through the Fast-track Research Funding Program to support publication in the top journal (Grant no. 42-FTTJ-70).

\section{References}

[1] S. Ruan and W. Wang, "Dynamical behavior of an epidemic model with a nonlinear incidence rate," Journal of Differential Equations, vol. 188, no. 1, pp. 135-163, 2003.

[2] A. Korobeinikov, "Global properties of infectious disease models with nonlinear incidence," Bulletin of Mathematical Biology, vol. 69, no. 6, pp. 1871-1886, 2007.

[3] M. De la Sen and S. Alonso-Quesada, "Vaccination strategies based on feedback control techniques for a general SEIRepidemic model," Applied Mathematics and Computation, vol. 218, no. 7, pp. 3888-3904, 2011.

[4] M. De la Sen, R. P. Agarwal, A. Ibeas, and S. Alonso-Quesada, "On a generalized time-varying SEIR epidemic model with mixed point and distributed time-varying delays and combined regular and impulsive vaccination controls," Advances in Difference Equations, vol. 2010, 42 pages, 2010.
[5] A. Korobeinikov, "Global properties of basic virus dynamics models," Bulletin of Mathematical Biology, vol. 66, no. 4, pp. 879-883, 2004.

[6] M. O. Souza and J. P. Zubelli, "Global stability for a class of virus models with cytotoxic $\mathrm{T}$ lymphocyte immune response and antigenic variation," Bulletin of Mathematical Biology, vol. 73, no. 3, article 609625, 2011.

[7] H. F. Huo, S. J. Dang, and Y. N. Li, "Stability of a two-strain tuberculosis model with general contact rate," Abstract and Applied Analysis, vol. 2010, 31 pages, 2010.

[8] C. Castillo-Chavez and B. Song, "Dynamical models of tuberculosis and their applications," Mathematical Biosciences and Engineering, vol. 1, no. 2, pp. 361-404, 2004.

[9] H. F. Huo and L. X. Feng, "Global stability for an HIV/AIDS epidemic model with different latent stages and treatment," Applied Mathematical Modelling, vol. 37, no. 3, pp. 14801489, 2013.

[10] R. Xu, "Global stability of an HIV-1 infection model with saturation infection and intracellular delay," Journal of Mathematical Analysis and Applications, vol. 375, no. 1, pp. 75-81, 2011.

[11] A. Yusuf, B. Acay, U. T. Mustapha, M. Inc, and D. Baleanu, "Mathematical modeling of pine wilt disease with Caputo fractional operator," Chaos, Solitons \& Fractals, vol. 143, article 110569, 2021.

[12] M. Arfan, H. Alrabaiah, M. Ur Rahman et al., "Investigation of fractal-fractional order model of COVID-19 in Pakistan under Atangana-Baleanu Caputo (ABC) derivative," Results in Physics, vol. 24, article 104046, 2021.

[13] C. Castillo-Garsow, G. Jordan-Salivia, and A. RodriguezHerrera, Mathematical Models for the Dynamics of Tobacco Use, Recovery and Relapse, 1997.

[14] G. Zaman, "Optimal campaign in the smoking dynamics," Computational and Mathematical Methods in Medicine, vol. 2011, 9 pages, 2011.

[15] G. Rahman, R. P. Agarwal, and Q. Din, "Mathematical analysis of giving up smoking model via harmonic mean type incidence rate," Applied Mathematics and Computation, vol. 354, pp. 128-148, 2019.

[16] M. Goyal, H. M. Baskonus, and A. Prakash, “An efficient technique for a time fractional model of Lassa hemorrhagic fever spreading in pregnant women," European Physical Journal Plus, vol. 134, no. 10, pp. 1-10, 2019.

[17] W. Gao, P. Veeresha, D. G. Prakasha, H. M. Baskonus, and G. Yel, "New approach for the model describing the deathly disease in pregnant women using Mittag-Leffler function," Chaos, Solitons \& Fractals, vol. 134, article 109696, 2020.

[18] R. Magin, Fractional Calculus in Bioengineering, Begell House Publishers, 2004.

[19] K. Shah, M. A. Alqudah, F. Jarad, and T. Abdeljawad, "Semianalytical study of Pine Wilt Disease model with convex rate under Caputo-Febrizio fractional order derivative," Chaos, Solitons \& Fractals, vol. 135, p. 109754, 2020.

[20] A. M. S. Mahdy, N. H. Sweilam, and M. Higazy, "Approximate solution for solving nonlinear fractional order smoking model," Alexandria Engineering Journal, vol. 59, no. 2, pp. 739-752, 2020.

[21] J. Singh, D. Kumar, M. Al Qurashi, and D. Baleanu, "A new fractional model for giving up smoking dynamics," Advances in Difference Equations, vol. 2017, no. 1, p. 16, 2017. 
[22] S. A. Khan, K. Shah, G. Zaman, and F. Jarad, "Existence theory and numerical solutions to smoking model under CaputoFabrizio fractional derivative," Chaos, vol. 29, no. 1, article 013128, 2019.

[23] A. M. S. Mahdy, M. S. Mohamed, K. A. Gepreel, A. AL-Amiri, and M. Higazy, "Dynamical characteristics and signal flow graph of nonlinear fractional smoking mathematical model," Chaos, Solitons \& Fractals, vol. 141, article 110308, 2020.

[24] H. Alrabaiah, A. Zeb, E. Alzahrani, and K. Shah, "Dynamical analysis of fractional-order tobacco smoking model containing snuffing class," Alexandria Engineering Journal, vol. 60, no. 4, pp. 3669-3678, 2021.

[25] A. A. Kilbas, O. I. Marichev, and S. G. Samko, Fractional Integrals and Derivatives: Theory and Applications, Gordon and Breach Switzerland, 1993.

[26] K. S. Miller and B. Ross, An Introduction to the Fractional Calculus and Fractional Differential Equations, Wiley, New York, 1993.

[27] A. A. Kilbas, H. Srivastava, and J. Trujillo, Theory and Application of Fractional Dierential Equations, vol. 204, Elseveir, North Holand, 2006.

[28] P. Kumar, V. S. Erturk, and M. Murillo-Arcila, "A complex fractional mathematical modeling for the love story of Layla and Majnun," Chaos, Solitons \& Fractals, vol. 150, article 111091, 2021.

[29] R. A. Khan and K. Shah, "Existence and uniqueness of solutions to fractional order multi-point boundary value problems," Communications in Applied Analysis, vol. 19, pp. 515-526, 2015.

[30] F. Awawdeh, A. Adawi, and Z. Mustafa, "Solutions of the SIR models of epidemics using HAM," Chaos, Solitons \& Fractals, vol. 42, no. 5, pp. 3047-3052, 2009.

[31] J. Biazar, "Solution of the epidemic model by Adomian decomposition method," Applied Mathematics and Computation, vol. 173, no. 2, pp. 1101-1106, 2006.

[32] A. Abdilraze, Admoian Decomposition Method: Convergence Analysis and Numerical Approximations, M.sc. Dissertation, McMaster University Hamilton, Canada, 2008.

[33] I. Ullah, S. Ahmad, M. Rahman, and M. Arfan, "Investigation of fractional order tuberculosis (TB) model via Caputo derivative," Chaos, Solitons \& Fractals, vol. 142, article 110479, 2021.

[34] F. Haq, K. Shah, G. Rahman, and M. Shahzad, "Numerical solution of fractional order smoking model via Laplace Adomian decomposition method," Alexandria Engineering Journal, vol. 57, no. 2, pp. 1061-1069, 2018.

[35] M. u. Rahman, M. Arfan, Z. Shah, P. Kumam, and M. Shutaywi, "Nonlinear fractional mathematical model of tuberculosis (TB) disease with incomplete treatment under Atangana-Baleanu derivative," Alexandria Engineering Journal, vol. 60, no. 3, pp. 2845-2856, 2021.

[36] A. Khan, T. S. Khan, M. I. Syam, and H. Khan, "Analytical solutions of time-fractional wave equation by double Laplace transform method," The European Physical Journal Plus, vol. 134, no. 4, p. 163, 2019.

[37] A. Kumar, S. Kumar, and S. Yan, "Residual power series method for fractional diffusion equations," Fundamenta Informaticae, vol. 151, no. 1-4, pp. 213-230, 2017.

[38] M. Kaplan and B. Ahmet, "A novel analytical method for timefractional differential equations," Optik, vol. 127, no. 20, pp. 8209-8214, 2016.
[39] M. Caputo and M. Fabrizio, "A new definition of fractional derivative without singular kernel," Progress in Fractional Differentiation and Applications, vol. 1, no. 2, pp. 1-13, 2015.

[40] A. Atangana and D. Baleanu, "New fractional derivatives with nonlocal and non-singular kernel: theory and application to heat transfer model," Thermal Science, vol. 20, no. 2, pp. 763-769, 2016

[41] M. Toufik and A. Atangana, "New numerical approximation of fractional derivative with non-local and non-singular kernel: application to chaotic models," The European Physical Journal Plus, vol. 132, no. 10, p. 444, 2017.

[42] J. Wang, L. Lv, and W. Zhou, "Ulam stability and data dependence for fractional differential equations with Caputo derivative," Electronic Journal of Qualitative Theory of Differential Equations, vol. 63, no. 63, pp. 1-10, 2011.

[43] D. Baleanu, J. T. Machado, and A. C. J. Luo, Fractional Dynamics and Control, Springer Science \& Business Media, 2011.

[44] D. Baleanu, D. Kai, and S. Enrico, Fractional Calculus: Models and Numerical Methods, World Scientific, 2012.

[45] S. Z. Rida, A. S. Abdel Rady, A. A. M. Arafa, and M. Khalil, "Approximate analytical solution of the fractional epidemic model," International Journal of Applied Mathematical Research, vol. 1, no. 1, pp. 17-19, 2012.

[46] F. B. M. Duarte and J. A. Tenreiro Machado, "Chaotic phenomena and fractional-order dynamics in the trajectory control of redundant manipulators," Nonlinear Dynamics, vol. 29, pp. 342-362, 2002.

[47] A. Atangana, "Fractal-fractional differentiation and integration: connecting fractal calculus and fractional calculus to predict complex system," Chaos, Solitons \& Fractals, vol. 102, pp. 396-406, 2017.

[48] A. S. Salem and B. S. T. Alkahtani, "Asymptotic analysis of a giving up smoking model with relapse and harmonic mean type incidence rate," Results in Physics, vol. 21, article 104437, 2021.

[49] K. A. Abro, "Role of fractal-fractional derivative on ferromagnetic fluid via fractal Laplace transform: a first problem via fractal-fractional differential operator," European Journal of Mechanics - B/Fluids, vol. 85, pp. 76-81, 2021.

[50] W. Chen, X. D. Zhang, and D. Korosak, "Investigation on fractional and fractal derivative relaxation- oscillation models," International Journal of Nonlinear Sciences and Numerical Simulation, vol. 11, no. 1, pp. 3-10, 2010.

[51] M. H. Heydari, "Numerical solution of nonlinear 2D optimal control problems generated by Atangana-Riemann-Liouville fractal-fractional derivative," Applied Numerical Mathematics, vol. 150, pp. 507-518, 2020.

[52] H. F. Huo and C. C. Zhu, "Influence of relapse in a giving up smoking model," Abstract and Applied Analysis, vol. 2013, 11 pages, 2013.

[53] R. Qesmi, J. Wu, J. Wu, and J. M. Heffernan, "Influence of backward bifurcation in a model of hepatitis B and C viruses," Mathematical Biosciences, vol. 224, no. 2, pp. 118-125, 2010.

[54] A. Granas and J. Dugundji, Fixed Point Theory, Springer, New York, 2005.

[55] Y. Başc1, S. Öğrekçi, and A. Mısır, “On Hyers-Ulam stability for fractional differential equations including the new Caputo-Fabrizio fractional derivative," Mediterranean Journal of Mathematics, vol. 16, no. 5, pp. 1-14, 2019. 
[56] J. Wang, K. Shah, and A. Ali, "Existence and Hyers-Ulam stability of fractional nonlinear impulsive switched coupled evolution equations," Mathematical Methods in the Applied Sciences, vol. 41, no. 6, pp. 2392-2402, 2018.

[57] K. Shah, A. Ali, and S. Bushnaq, "Hyers-Ulam stability analysis to implicit Cauchy problem of fractional differential equations with impulsive conditions," Mathematical Methods in the Applied Sciences, vol. 41, no. 17, pp. 8329-8343, 2018. 